DOI: https://doi.org/10.24867/06BE25Pocuca

\title{
PREDLOG ARHITEKTURE CI/CD PIPELINE-A KORIŠĆENJEM JENKINS I DOCKER ALATA
}

\section{THE PROPOSAL OF CI/CD PIPELINE USING JENKINS AND DOCKER}

\author{
Milena Počuča, Srđan Popov, Fakultet tehničkih nauka, Novi Sad
}

\begin{abstract}
Oblast - ELEKTROTEHNIKA I RAČUNARSTVO
Kratak sadržaj - U radu su detaljno analizirana dva najpopulanija alata za pravljenje CI/CD pipeline-a. Infrastruktura je postavljena uz akcenat na Docker na kome su pokrenuti svi servisi potrebni za CI/CD. Za primer aplikacije na kojoj je pipeline implementiran uzeta je SpringBoot $i$ AngularJS aplikacija podsistema za fakturisanje.
\end{abstract}

Ključne reči: $C I / C D$, Docker, Jenkins, SpringBoot, AngularJS, pipeline, DevOps

Abstract - The paper analyzes the two most popular tools for implementing CI/CD pipeline. The infrastructure is set up with the accent on Docker on which all the services neeeded for this pipeline are configured and run. The application on which the pipeline was implemented is a SpringBoot and AngularJS application subsystem for invoices.

Keywords: CI/CD, Docker, Jenkins, SpringBoot, AngularJS, pipeline, DevOps

\section{UVOD}

CI/CD predstavlja skup CI (continuous integration) i CD (continuous delivery) paradigmi koji je našao široku primenu $\mathrm{u}$ industriji. CI predstavlja pristup $\mathrm{u}$ kome se programeri podstiču da implementiraju male izmene na kodu što je frekventnije moguće. CD se nadovezuje na CI i automatizuje proces isporuke aplikacija na izabrana okruženja. CI/CD pipeline u radu je implementiran korišćenjem Jenkins i Docker alata. CI/CD pipeline-om bavi se DevOps.

Cilj rada je detaljno upoznavanje sa Jenkins i Docker alatima uz demonstraciju načina implementacije infrastrukture za CI/CD, kao i uz osvrt na već postojeće implementacije pipeline-a.

\section{INFRASTRUKTURA SISTEMA}

Jezgro sistema predstavlja Docker mašina koja u sebi ima pokrenute kontejnere svih alata koji su potrebni za funkcionisanje aplikacije. Na Docker-u su startovani na lokalnom računaru Jenkins kao CI server, GitLab kao sistem za upravljanje git repozitorijumom, Minio namenjen čuvanju biblioteka kreiranih od strane Jenkins-a, MySQL Server za upravljanje bazom podataka, SonarQube za proveru kvaliteta koda, Redis za konfiguraciju Docker registra.

\section{NAPOMENA:}

Ovaj rad proistekao je iz master rada čiji mentor je bio dr Srđan Popov, vanr. prof.
$\mathrm{Na}$ slici 1 prikazan je primer arhitekture sistema koji se bazira na Docker-u.

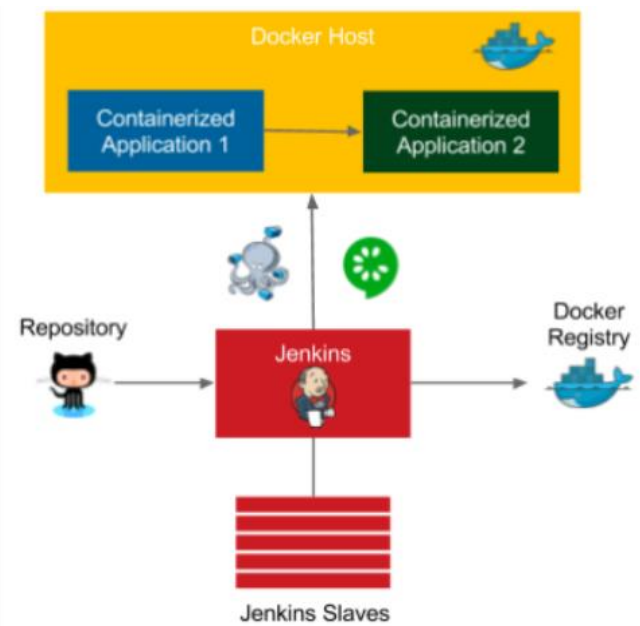

Slika 1. Prikaz arhitekture bazirane na Docker-u

\section{DEVOPS}

DevOps predstavlja spoj tehnologija koje objedinjuju razvoj softvera (Dev) i IT operacija $(O p s)$ kako bi skratili životni ciklus razvoja softvera.

Ciljevi DevOps paradigme su učestalije isporučivanje koda, skraćivanje vremena potrebnog da se stigne do produkcionog rešenja kao i vremena koje protekne od kreiranja zahteva do njihovog ispunjenja, snižavanje stope otkaza novijih verzija proizvoda, brže srednje vreme oporavka sistema.

\section{JENKINS}

Jenkins je samostalan, javni automatizacioni server koji se može koristi da automatizuje razne vrste zadataka povezane sa izgradnjom, testiranjem, isporukom ili deployment-om softvera. Jedna od najvećih prednosti ovog servera je velika proširivost koja omogućava da se njegove funkcionalnosti prošire instalacijom plugin-ova.

\subsection{Pipeline}

Jenkins pipeline je skup plugin-ova koji omogućava implementaciju i integraciju continuous delivery pipelineova u Jenkins. Jenkinsfile komitovan na repozitorijum omogućava veliki broj prednosti jer je verzionisan i u svakom trenutku su sve izmene na fajlu vidljive.

\subsection{Zašto pipeline}

Podržava širok skup slučajeva od jednostavih continuous integration do obimnih CD pipeline-ova. Sadrži brojne funkcionalnosti, kao što su implementacija pipeline-a $\mathrm{u}$ kodu, pauziranje, svestranost, proširivost. 


\subsection{Rečnik pojmova}

Osnovni pojmovi u Jenkins-u su: agent, artifact, build, core, downstram, executor, fingerprint, folder, item, job, label, master, node, project, pipeline, plugin, stage, step, trigger [4].

\section{SINTAKSA PIPELINE-A}

Od verzije 2.5 plugin-a za pipeline, postoje dve sintakse za njen opis koje će u nastavku biti objašnjene.

\subsection{Deklarativni pipeline}

Ova sintaksa je prilično nov dodatak Jenkins pipeline-a koji predstavlja dosta jednostavniji način definisanja. Svaki pipeline ovog tipa mora biti obuhvaćen u okviru pipeline bloka.

\subsubsection{Sekcije}

Obično su sastavljene iz jedne ili više direktiva.

\subsubsection{Agent}

Sekcija agent određuje gde će se čitav pipeline, ili određena faza, izvršavati na Jenkins okruženju, u zavisnosti od toga gde je sekcija agent smeštena. Mora se definisati na najvišem nivou unutar pipeline bloka, ali se može definisati i unutar stage bloka. Sadrži parametre koji se mogu primeniti na najviši nivo pipeline bloka, ili unutar svake stage direktive. Mogući parametri su any, none, label, node, docker, dockerfile, kubernetes [4].

\subsubsection{Post}

Ova sekcija sadrži jedan ili više dodatnih koraka koji se pokreću nakon završetka run dela pipeline-a ili stage-a. Podržava brojne post-condition blokove koji dozvoljavaju izvršavanje koraka unutar svakog uslova. Mogući uslovi su: always, changed, fixed, regression, aborted, failure, success, unstable, unsuccessful, cleanup [4].

\subsubsection{Stages}

Ova sekcija sadrži sekvencu jedne ili više stages direktiva i označava sekciju gde je najveći deo posla u pipeline-u smešten. Preporučuje se da ova sekcija sadrži bar jednu stage direktivu za svaki deo CD procesa - Build, Test, Deploy [4].

\subsubsection{Steps}

Sekcija steps definiše skup jednog ili više koraka koji se mogu izvršavati u datoj stage direktivi. Sekcija je obavezna, ne zahteva parametre i dozvoljena je unutar svakog stage bloka.

\subsubsection{Direktive}

\subsubsection{1. environment}

Ova direktiva predstavlja sekvencu ključ-vrednost parova koji će biti definisani kao varijable okruženja za sve korake, ili za korake specifične za neki stage, u zavisnosti od toga gde se ova direktiva nalazi u pipeline-u. Podržani tipovi kredencijala: Secret Text, Secret File, Username and Password, SSH with Private key [4].

\subsubsection{2. options}

Direktiva options omogućuje konfiguraciju opcija karakterističnih za pipeline unutar samog pipeline-a. Dostupne opcije za pipeline: buildDiscarder, checkoutTo-Subdirectory, disableConcurrentBuilds, disableResume, newContainerPerStage. overrideIndexTriggers, quiet-Period, retry, skipDefaultCheckout, skipStagesAfter-Unstable, timeout, timestamps, parallelsAlwaysFailFast, Opcije koje se odno- se na stage predstavljaju deo opcija za pipeline:skipDefaultCheckout, timeout, retry, timestamps [4].

\subsubsection{3. parameters}

Direktiva parameters obezbeđuje listu parametara koji bi korisnik trebalo da navede kada pokreće izvršavanje pipeline-a. Tipovi parametara su: string, text, booleanParam, choice, password.

\subsubsection{4. triggers}

Direktiva triggers definiše automatski način na koji bi pipeline trebalo da se ponovo trigeruje. Vrste trigera su: cron, pollSCM, upstream [4].

\subsubsection{Jenkins cron sintaksa}

Jenkins cron sintaksa prati sintaksu cron utility-ja sa sitnim razlikama. Svaka linija se sastoji iz 5 delova koji su odvojeni TAB-om ili razmakom. U sintaksi se specificiraju ' $M$ H DOM MONTH DOW', gde $M$ predstavlja minut $\mathrm{u}$ satu, $\mathrm{H}$ sat $\mathrm{u}$ danu, DOM dan $\mathrm{u}$ mesecu, MONTH mesec u godini, a DOW dan u nedelji.

\subsubsection{5. stage}

Direktiva stage se nalazi u okviru stages sekcije. Sadrži steps sekciju, opcionu agent sekciju ili druge direktive specifične za stage. U praksi, najveći deo realnog posla pipeline-a se odvija unutar jedne ili više stage direktiva. Potrebno je definisati bar jednu stage direktivu koja će imati jedan obavezni parameter, a to je ime stage-a.

\subsubsection{6. tools}

Ova sekcija definiše alate koji se automatski instaliraju i dodaju na PATH. Ignoriše se ukoliko se specificira agent none. Dozvoljena je unutar pipeline ili stage bloka. Podržani alati su maven, jdk i gradle.

\subsubsection{7 when}

Direkvita when dozvoljava pipeline-u da odluči da li stage treba da se izvrši u zavisnosti od datog uslova. Unapred dostupni uslovi su: branch, buidlingTag, changelog, changeset, changeRequest. environment, equals, expression, tag, not, allOf, anyOf, triggeredBy [4].

\subsubsection{Steps}

Deklarativni pipeline koriste sve korake koji su dokumentovani u Pipeline Steps reference dokumentaciji i sadrže obilnu listu koraka.

\subsection{Skriptovani pipeline}

Za razliku od deklarativnog, skriptovani pipeline je DSL opšte namene izgrađen pomoću Groovy-ja. Većinu fukncionalnosti koje omoguće Groovy jezik moguće je koristiti u skriptovanom pipeline-u. Najvažniji aspekt ovog pipeline-a je kontrola toka.

\subsection{Deklarativni naspram skriptovanog pipeline-a}

Oba pipeline-a su fundamentalno ista $\mathrm{u}$ pipeline podsistemu. Razlikuju se ipak, u sintaksi i fleksibilnosti. Deklarativni pipeline ograničava šta je dostupno korisniku sa striktnijom i predefinisanom strukturom, što ga čini idealnim izborom za jednostavniji CD pipeline. Skriptovani pipeline obezbeđuje mali broj ograničenja, može se reći da su to ograničenja definisana u samom Groovy jeziku, ne u pipeline-u, što ga čini idealnim izborom za iskusnije korsnike i korisnike koji imaju kompleksne zahteve. Deklarativni pipeline promoviše deklarativni model programiranja, dok skriptovani pipeline promoviše imperativni [4]. 


\section{DOCKER}

Docker je alat koji je dizajniran da olakša kreiranje, deploy-ovanje i pokretanje aplikacija korišćenjem kontejnera. Docker, Inc. je osnovan 2010. godine, a javnosti je predstavljen 2013.

\subsection{Kontejneri i virtuelne mašine}

Docker kontejneri se mogu porediti sa virtuelnim mašinama. I jedni i drugi imaju sličan cilj, a to je izolacija aplikacije i njenih zavisnosti u samoodrživu jedinicu koja se može svuda pokrenuti. Virtuelna mašina ima svoj virtuelni operativni sistem u kome hipervizor igra esencijalnu ulogu tako što joj obezbeđuje platformu za upravljanje i izvršavanje guest operativnog sistema.

$\mathrm{Za}$ razliku od virtuelne mašine, kontejner obezbeđuje virtuelizaciju na nivou operativnog sistema. Kontejnerizacija je takođe vrsta virtualizacije s prednošću da je efikasnija jer nema guest operativnog sistema, već koristi host-ov operativni sistem.

\subsection{Docker Engine}

Docker engine predstavlja srž Dockera. To je aplikacija koja se instalira na host mašinu. Funkcioniše po principu klijent-server arhitekture. Glavne komponente ove arhitekture su: server koji predstavlja program koji se dugo izvršava zvani daemon proces, CLI koji prestavlja klijenta, REST API preko koga CLI i daemon komuniciraju [5].

\subsection{Arhitektura Docker-a}

Docker koristi klijent-server arhitekturu u kojoj Docker klijent komunicira sa Docker daemon-om koji ustvari radi najveći posao - izgradnju, pokretanje i distribuciju Docker kontejnera.

\subsection{Slojevi Docker-a}

Docker image se kreira iz serije slojeva (eng. layers) gde svaki sloj predstavlja instrukciju image-a u Dockerfile-u. Svi slojevi sem poslednjeg sloja imaju samo pristup čitanja, dok se na poslednjem sloju može i pisati.

\subsection{Dockerfile}

Docker automatski pravi image čitanjem instrukcija iz Dockerfile-a. Ovaj fajl je tekstualni dokument koje korisnik može da koristi da bi napravio image. Komanda docker build pravi image.

Dostupne instrukcije koje se zadaju su:

FROM (inicijalizuje build i postavlja bazni image za ostale instrukcije),

$R U N$ (izvršava komande u novom sloju koji je iznad image-a),

EXPOSE (obaveštava Docker-a da kontejner sluša na određenom portu),

$E N V$ (postavlja varijablu na zadatu vrednost),

$A D D$ (kopira nove fajlove iz zadatog izvora i dodaje iz na fajl sistem image-a),

COPY (kopira nove fajlove iz izvora i dodaje ih na fajl sistem kontejnera),

VOLUME (kreira pristupnu tačku sa zadatim imenom i pamti je kao eksterni volumen host-a ili drugih kontejnera), USER (postavlja korisniko ime i grupu korisnika koji će se koristiti za pokretanje image-a) [5].

\subsection{Docker compose}

Compose je alat za definisanje i pokretanje Docker aplikacija sa više kontejnera. Koristi yaml fajl za konfigurisanje servisa aplikacije. To je proces koji se sastoji iz tri kora- ka: definisanje okruženja aplikacije korišćenjem Dockerfile-a, definisanje servisa koji prave aplikaciju u dockercompose.yml fajlu i pokretanje docker-compose up komande kako bi se sve to pokrenulo u izolovanom okruženju [5].

\section{ALATI ZA IZGRADNJU CI/CD PIPELINE-A}

$\mathrm{CI} / \mathrm{CD}$ pipeline se može postaviti korišćenjem većeg broja alata. Ovo predstavlja trenutno najpopularniji trend $\mathrm{u}$ razvoju softvera.

\subsection{Jenkins}

Ovo je najpopularnjie rešenje $\mathrm{CI} / \mathrm{CD}$ pipeline-a koje je besplatno.

\subsection{Microsoft VSTS}

VSTS nije samo servis za CI/CD, već i repozirotijum za kod i planiranje projekata. VSTS je pravljen za Visual Studio, ali se može integrisati i sa drugim alatima. Besplatan je za timove do 5 članova.

\subsection{Bamboo (Atlassian Dev Tool)}

Bamboo je CI server koji je Atlassian-ov proizvod, podržava Docker, ima probni period od 30 dana, nakon čega se naplaćuje.

\subsection{GitLab}

GitLab predstavlja skup alata za upravljanje skoro svim aspektima životnog ciklusa razvoja softvera. Dostupan je kao besplatan Community i Enterprise edicija koja može biti host-ovana ili se nalaziti na mašini.

\subsection{Codeship}

U odnosu na druge alate koji pružaju UI za naprednu konfiguraciju, Codeship je uglavnom baziran na skriptama. Poseduje Basic i Pro verzije koje se naplaćuju.

\subsection{Codefresh}

Codefresh svaki korak u pipeline-u izvršava na posebnom kontejneru. Besplatna verzija omogućuje 3 korisnika mesečno, Pro verzija 10, dok Enterprise omogućuje neograničen broj.

\subsection{TeamCity}

TeamCity je CI server koji ima ugrađenu integraciju sa Docker-om. Dostupan je besplatno sa ograničenom licencom, a postoji i verzija koja se plaća.

\subsection{Travis CI}

Travis CI je host-ovani servis za GitHub projekte. Besplatan je za javne projekte i omogućuje pretplatu za privatne projekte.

\subsection{GoCD}

GoCD je besplatan softver koji se instalira lokalno. Ima i plugin za docker, besplatan je uz opciju plaćanja podrške.

\subsection{CircleCI}

CircleCI je drugačiji od ostalih alata jer podržava buildovanje, testiranje i deploy-ovanje iOS i macOS projekata korišćenjem macOS virtuelne mašine, mada se može build-ovati i korišćenjem Linux virtuelne mašine. Cena CicrleCI-a zavisi od kontejnera i platforme. Linux kontejner je besplatan, ali macOS kontejneri i lokalna instalacija se plaćaju. 


\section{OSVRT NA POSTOJEĆE IMPLEMENTACIJE CI/CD PIPELINE-A}

\subsection{Automatizacija CI/CD pipeline-a za projekat baziran na agilnom pristupu}

Ciljevi ovog rada su razmevanje CI/CD pipeline-a analizom postojećih metoda, pronalaženje odgovarajuće metode za merenje performansi postojećeg sistema, predlog mehanizma za izvršavanje testova opterećenja korišćenjem saobraćaja u produkciji sa minimalnim posledicama na sistem. U radu su od alata korišćeni Git kao sistem za kontrolu verzija, Nexus kao repozitorijum izvršivih fajlova i Jenkins kao CI server. Zaključeno je da je CI/CD pipeline poboljšao agilan proces isporučivanja $\mathrm{i}$ poboljšao je produktivnost sistema [1].

\subsection{CI/CD za HPC korišćenjem Jenkins-a i Singularity-ja}

U radu je opisano kako je Research Computing ogranak Bolder (eng. Boudler) Univerziteta u Koloradu primenio CI/CD paradigmu na RMACC Summit supercomputer kako bi obezbedio sistem inženjerima i istraživačima da iskoriste prednosti CI/CD paradigme.

Kao CI server, korišćen je Jenkins koji je povezan sa Singularity-jem koji predstavlja rešenje za kontejnerizaciju. Uključivanjem $\mathrm{CI} / \mathrm{CD}$ paradigme u proces rada HPC-a je povećalo potencijal za isporuku visoko kvalitetnih i pouzdanih softvera [2].

\subsection{Implementacija CI principa u maloj kompaniji: studija slučaja}

Kao motivacioni faktori za razvoj ove paradigme u kompaniji, identifikovani su poboljšanje kvaliteta sotfvera, veća pouzdanost release-a, kraći time-to-market i poboljšanje produktivnosti programera. Centralizovano rešenje, kao što je GitLab je primećeno kao najprimerenije rešenje jer on spaja sve potrebne servise. Zaključeno je da je $\mathrm{CI} / \mathrm{CD}$ paradigma donela poboljšanje rada kompanije, ali da je potrebno nastaviti sa radom na istoj, tako što će preostale repozitorijume migrirati na novi sistem i početi što pre sa testiranjem [3].

\section{IMPLEMENTACIJA REŠENJA}

Čitav sistem počiva na Docker-u. U njemu su pokrenuti GitLab, Jenkins, Redis, SonarQube, Minio i MySQL. Sistem radi tako što kada se kod postavi na repozitorijum, Jenkins pipeline registruje da je došlo do izmene repozitorijuma, povlači novi kod i pokreće build aplikacije. Nakon što se build završi, startuju se testovi i ukoliko se svi uspešno izvrše, .jar koji je napravljen se postavlja na Minio i šalje se u Docker koji kreira novi kontejner za aplikaciju. SonarQube vrši analizu novog koda uz proveru pokrivenosti koda testovima. Kako Docker ponovo pokreće kontejner aplikacije sa novonastalim izmenama, njih je moguće odmah testirati.

\section{ZAKLJUČAK}

$\mathrm{U}$ radu je prikazano kako se konfiguriše $\mathrm{CI} / \mathrm{CD}$ pipeline za potrebe manjeg projekta uz detaljna objašnjena dva glavna korišćena alata - Docker i Jenkins. Neosporivo je da je CI/CD paradigma izuzetno značajna za razvoj IT kompanija sa svim prednostima koje donosi.

Postoji veliki broj alata za implementaciju ove paradigme, pa treba dobro proučiti kada koji iskoristiti, Jenkins predstavlja vodeći alat danas sa svim svojim pluginovima i novim deklarativnim pipeline-om. Docker je takođe vodeći alat jer ima podršku za Kubernetes koji upravlja kontejnerima na distribuiranim sistemima. Dalji razvoj arhitekture bi uključio Kubernetes i distribuciju sistema.

\section{LITERATURA}

[1] S.A.I.B.S. Arachici, Indika Perera, „Continuous Integration and Continuous Delivery Pipeline Automtion for Agile Software Poject Management“, Department of Computer Science and Engineering, University of Moratuwa. Moratuwa, Sri Lanka

[2] Zebula Sampedro, Aaron Holt, Thomas Hauser, „Continuous Integration and Delivery for HPC: Using Singularity and Jenkins“, Univeristy of Colorado Boulder, Research Computing, Boulder, Colorado

[3] Kim Rejström, „Implementing Continuous Integration in a Small Company: A Case Study“", Aalto University, School of Science

[4] https://jenkins.io/doc/ (pristupljeno u septembru 2019.)

[5] https://docs.docker.com (pristupljeno u septembru 2019.)

\section{Kratka biografija:}

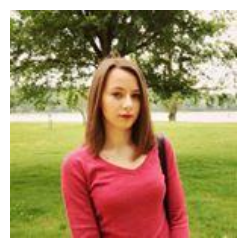

Milena Počuča, rođena je 27.08.1994. u Kraljevu. 2013. godine upisuje „Fakultet tehničkih nauka" u Novom Sadu, smer „Računarstvo i automatika”. 2017. godine dobija zvanje Diplomirani inženjer elektrotehnike i računarstva. 2017/18. godine upisuje master akademske studije, smer „Primenjene računarske nauke i informatika - Elektronsko poslovanje”. 01.03.2018. godine dobija zvanje Saradnika u nastavi na Departmanu za računarstvo i automatiku na Fakultetu tehničkih nauka u Novom Sadu. kontakt: minapocuca@gmail.com 